
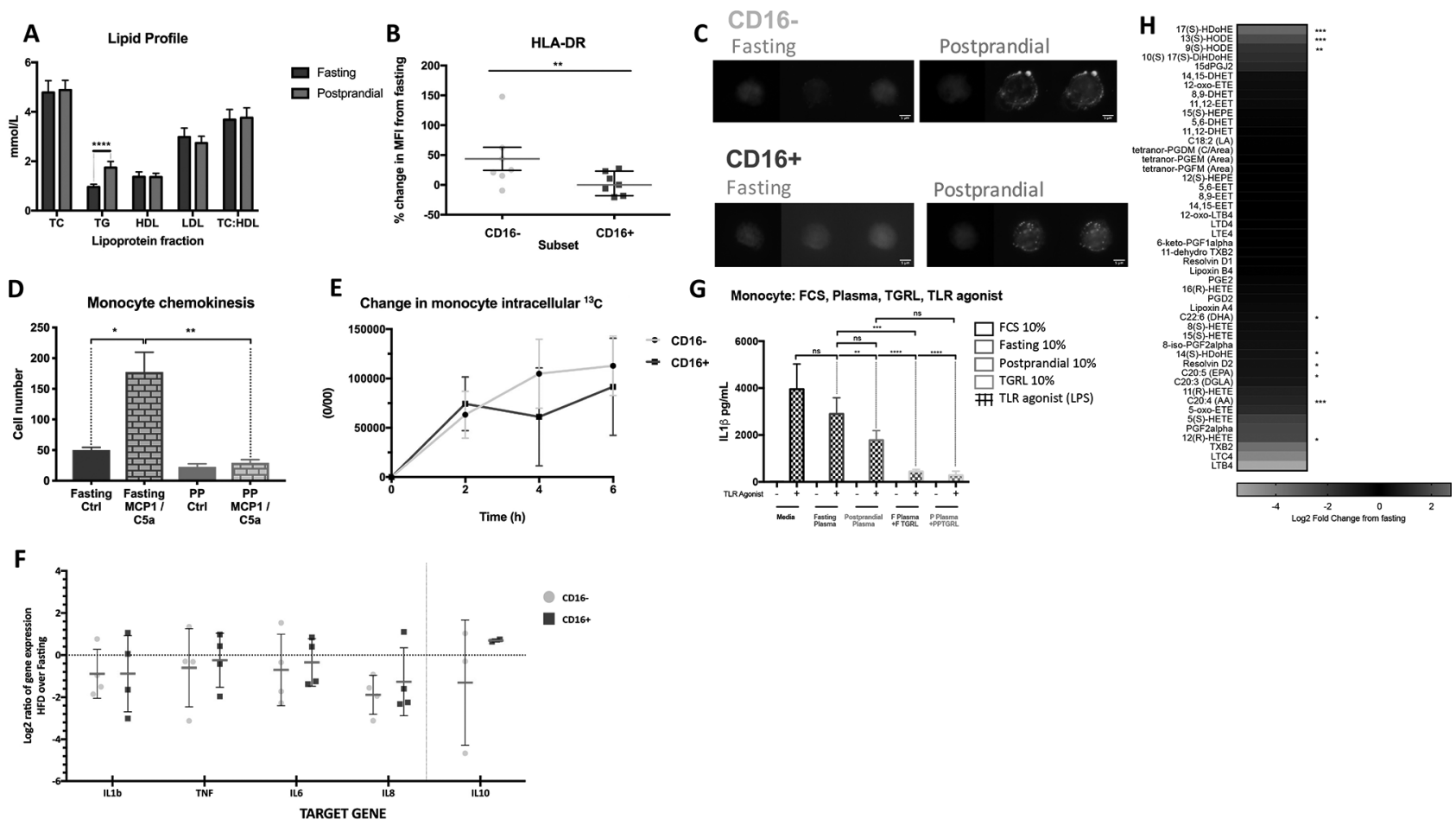

Abstract BS30 Figure 1 A) Significant increase in the TG lipoprotein fraction at 4h postprandial. B) Increase in HLA-DR expression in postprandial CD16-(classical) monocytes (assessed by FACS). C) CD16-monocytes preferentially accumulate neutral intracellular lipid droplets ex vivo when comparing monocytes purified before (fasting) and 4hours following (postprandial) a SF-rich meal. Nucleus stained with DAPI (blue) and lipid stained with LipidTox (greens) stains. Representative images shown. D) Postprandial monocytes (both subsets)reduce ability to migrate towards a MCP/C5a chemokine gradient when lipid loaded in postprandial state. E) Monocytes accumlate exogenous ${ }^{13} \mathrm{C}$-palmitic with subsets showing similar kinetics. F) Monocyte inflammatory cytokine release (IL1? shown) is attenuated in vitro after incubation in media supplementated with postprandial plasma and increased in effect with postprandial TGRL after TLR agonist stimulation with LPS. G) Monocyte subset inflammatory expression profile demonstrate no significant upregulation of inflammatory genes and downregulation of IL8. H) Heatmap demonstrating log2 fold changes in intracellular lipid accumulation in postprandial monocytes. ${ }^{*} \mathrm{P} \leq 0.05,{ }^{*} \mathrm{P} \leq 0.01,{ }^{* *} \mathrm{P} \leq 0.001$.

Abbreviations:TG = triglyceride,$H L A=$ human leukocyte antigen, FACS = fluorescence activated cell sorting, FCS = fetal calf serum, $M C P=$ monocyte chemoattractant protein, C5a = complement component 5a, IL = interleukin, TGRL = TG rich lipoprotein, TLR = toll-like receptor.

\section{BS31 THE ROLE OF FRACTALKINE AND CX3CR1-EXPRESSING LYMPHOCYTES DURING MYOCARDIAL ISCHAEMIA/ REPERFUSION INJURY}

${ }^{1}$ Lilia Draganova*, ${ }^{2}$ Rachael Redgrave, ${ }^{1}$ Simon Tual-Chalot, ${ }^{1}$ Sarah Marsh, ${ }^{1}$ Helen Arthur, ${ }^{2}$ loakim Spyridopoulos. 'Newcastle University; ${ }^{2}$ Institute of Genetic Medicine, Newcastle University

\subsection{6/heartjnl-2019-BCS.194}

Introduction Primary percutaneous coronary intervention (PPCI) is the standard care for treatment of acute myocardial infarction, reducing both mortality and morbidity. However, ischaemia/reperfusion ( $/ / R)$ injury remains an important complication, contributing up to $50 \%$ of the final infarct size. Evidence from our clinical studies suggests that lymphocytes expressing the fractalkine receptor (CX3CR1) are associated with microvascular obstruction and hence poorer long-term patient outcomes. This project aims to investigate the role of fractalkine in lymphocyte-mediated myocardial $\mathrm{I} / \mathrm{R}$ injury and evaluate whether this damage can be reduced by inhibiting fractalkine/receptor (CX3CL1/CX3CR1) interaction.

Methods We are using a mouse model of myocardial $\mathrm{I} / \mathrm{R}$ injury to study lymphocyte infiltration following MI. Multicolour flow cytometry, immunofluorescence staining, qPCR, imaging mass cytometry and magnetic resonance imaging (MRI) are used to evaluate $\mathrm{T}$ cell recruitment and cardiac function in a CX3CR1 knockout mouse line.
Results CX3CR1 knockout leads to over 30\% reduction in immune cell infiltration at the site of cardiac ischaemic injury 2 hours after reperfusion, both in heterozygous $(\mathrm{p} \leq 0.01)$ and knockout $(\mathrm{p} \leq 0.001)$ mice. By 24 hours $\mathrm{I} / \mathrm{R}$, infiltration of CD45 + cells in heterozygous and knockout animals returns to, and surpasses, wild type (WT) levels ( $\mathrm{p} \leq 0.05$ ). Despite the $20 \%$ increase in leukocyte infiltration at 24 hours, we see a 2-fold decrease in $\mathrm{T}$ cells at the site of $\mathrm{I} / \mathrm{R}$ injury in the knockout animals, compared to both WT $(\mathrm{p} \leq 0.001)$ and heterozygous mice $(\mathrm{p} \leq 0.0001)$. T cell infiltration in the knockout is also reduced (although not significantly) when heart infiltrates at $24 \mathrm{~h}$ reperfusion are investigated by an alternative method: flow cytometry analysis of digested cardiac tissue. Furthermore, our studies show a reduction of $30 \%$ in the number of CX3CR1-expressing T cells $(\mathrm{p} \leq 0.0001)$ and $50 \%$ in total CX3CR1+ cells $(\mathrm{p} \leq 0.01)$ that infiltrate the left ventricle wall in knockout mice, compared to heterozygous animals. Functional studies using cardiac MRI show no difference between heterozygous and $\mathrm{KO}$ mice 6 weeks following I/R injury.

Conclusion These findings suggest that knockout of CX3CR1 leads to delayed recruitment of leukocytes following cardiac I/ $\mathrm{R}$. This results in an altered immune cell infiltrate including reduced number of $\mathrm{T}$ cells in the injured tissue. In addition, the role for CX3CR1-expressing $\mathrm{T}$ cells in myocardial $\mathrm{I} / \mathrm{R}$ is supported by our data showing that the number of CX3CR1 